\title{
On Tangential Star Products for the Coadjoint Poisson Structure ${ }^{\star}$
}

\author{
M. Cahen ${ }^{1}$, S. Gutt ${ }^{1,2}$, J. Rawnsley ${ }^{3}$ \\ 1 Département de Mathématiques, Université Libre de Bruxelles, Campus Plaine CP 218, 1050 Bruxelles, \\ Belgium Email: mcahen@ulb ac be, sgutt@ulb ac be \\ 2 Département de Mathématiques, Université de Metz, Ile du Saulcy, F-57045 Metz cedex, France \\ Email: gutt@poncelet univ-metz fr \\ 3 Mathematics Institute, University of Warwick, Coventry CV4 7AL, UK \\ Email: j.rawnsley@warwick ac uk
}

Received: 24 November 1995/Accepted: 5 January 1996

\begin{abstract}
We derive necessary conditions on a Lie algebra from the existence of a star product on a neighbourhood of the origin in the dual of the Lie algebra for the coadjoint Poisson structure which is both differential and tangential to all the coadjoint orbits. In particular we show that when the Lie algebra is semisimple there are no differential and tangential star products on any neighbourhood of the origin in the dual of its Lie algebra.
\end{abstract}

\section{Introduction}

The notion of star-product on a Poisson manifold, introduced in $[1,2]$, has been extensively studied in the literature. The existence of star-products has been shown in complete generality in the case of symplectic manifolds using several different approaches. See $[5,6,11]$ and the references cited therein. Recently some progress has been made for Poisson manifolds in various special cases [6, 10, 12].

Any Poisson manifold is foliated by symplectic manifolds [14] called symplectic leaves and it is natural to ask if star-products can be constructed on Poisson manifolds by gluing together star-products on the symplectic leaves. Such a star-product we call tangential. In the case of regular Poisson manifolds where the leaves have constant dimension the symplectic methods extend to yield tangential star-products.

The simplest Poisson structures are those where the Poisson tensor is linear in coordinates and these are obtained as the dual of a Lie algebra (the structure constants are obtained from the Poisson brackets of linear functions). These Poisson tensors are not regular as they must vanish at the origin. The dual of the Lie algebra of $S U(2)$ has symplectic leaves which are round 2-spheres centered at the origin and so is regular outside the origin. We initially tried to see if we could choose Fedosov style starproducts on the leaves in a way which would extend over the origin. To be tangential

\footnotetext{
* Research partially supported by EC contract CHRX-CT920050 Correspondence to: J. Rawnsley
} 
at the origin would require the higher order terms to vanish there. We found this led already to a contradiction at the second order terms even if we allowed the choice of symplectic connection to vary from leaf to leaf.

In this paper we work from the opposite direction with a given Lie algebra by assuming that we have a tangential star-product on its dual and seeing what conditions are imposed on the Lie algebra by the smoothness of the second order terms.

We show that, when we have a tangential star-product on the dual of a Lie algebra, each invariant quadratic form on the dual forces an associated ideal to be two-step nilpotent. If the quadratic form is non-degenerate then the ideal is the whole Lie algebra. The proof uses only the existence of the star-product in a neighbourhood of the origin and only needs existence and associativity to second order. It essentially says that when a Lie algebra has a sufficiently complicated coadjoint orbit structure near the origin then it cannot also have a tangential star-product. In particular we obtain (Corollary 2):

Theorem 1. If $\mathfrak{g}$ is a semisimple Lie algebra there is no differential star-product on any neighbourhood of the origin in $\mathfrak{g}^{*}$ which is tangential to the coadjoint orbits.

Let $K$ be a compact semisimple Lie group and $K^{\mathbb{C}}=K A N$ a Iwasawa decomposition of its complexification. Lu and Weinstein [8] show how $K$ and $A N$ have Poisson structures which make them into Poisson Lie groups. The Poisson structure on $A N$ is locally isomorphic to that on $\mathfrak{k}^{*}$ in neighbourhoods of the identity and origin respectively. By Theorem $1 A N$ gives an example of a Poisson Lie group which does not have a differential star-product which is tangential to the symplectic leaves. We do not know if the same is true for the Poisson Lie structure on $K$. See Remark 6 for more information.

\section{Notation}

In what follows we employ the summation convention on pairs of upper and lower indices.

Let $G$ be a connected Lie group with Lie algebra $\mathfrak{g}$. If $X_{1}, \ldots, X_{n}$ is a basis for $\mathfrak{g}$ the structure constants $C_{i j}{ }^{k}$ are defined by

$$
\left[X_{i}, X_{j}\right]=C_{i j}^{k} X_{k}
$$

so $C_{i j}{ }^{k}=-C_{j i}{ }^{k}$ and the Jacobi Identity translates as

$$
C_{i j}{ }^{l} C_{l k}{ }^{m}+C_{j k}^{l} C_{l i}{ }^{m}+C_{k i}{ }^{l} C_{l j}{ }^{m}=0 .
$$

Let $\mathfrak{g}^{*}$ be the dual space of $\mathfrak{g}$ and $\xi^{1}, \ldots, \xi^{n}$ the dual basis so that $\left\langle\xi^{a}, X_{b}\right\rangle=\delta_{b}^{a}$. The basis determines linear coordinate functions $x_{1}, \ldots, x_{n}$ on $\mathfrak{g}^{*}$ by

$$
\xi=x_{a}(\xi) \xi^{a}, \quad x_{a}(\xi)=\left\langle\xi, X_{a}\right\rangle .
$$

$(g, \xi) \mapsto g \cdot \xi=\xi \circ \mathrm{Adg}^{-1}$ denotes the coadjoint action of $G$ on $\mathfrak{g}^{*}$ and this differentiates to give the action $X \cdot \xi=-\xi \circ \operatorname{adX}$ of $\mathfrak{g}$.

Let $\beta$ be a symmetric bilinear form on $\mathfrak{g}^{*}$ with associated quadratic form $\Delta(\xi)=$ $\beta(\xi, \xi)$. Then $\Delta=\beta^{a b} x_{a} x_{b}$, where $\beta^{a b}=\beta\left(\xi^{a}, \xi^{b}\right)$. $\beta$ defines a linear map $\widehat{\beta}: \mathfrak{g}^{*} \rightarrow \mathfrak{g}$ by 


$$
\langle\eta, \widehat{\beta}(\xi)\rangle=\beta(\xi, \eta), \quad \forall \xi, \eta \in \mathfrak{g}^{*} .
$$

We transfer the coadjoint action to bilinear forms by $(g \cdot \beta)(\xi, \eta)=\beta\left(g^{-1} \cdot \xi, g^{-1} \cdot \eta\right)$. Then

$$
\begin{aligned}
\langle\eta, \widehat{g \cdot \beta}(\xi)\rangle & =(g \cdot \beta)(\xi, \eta) \\
& =\beta\left(g^{-1} \cdot \xi, g^{-1} \cdot \eta\right) \\
& =\left\langle g^{-1} \cdot \eta, \widehat{\beta}\left(g^{-1} \cdot \xi\right)\right\rangle \\
& =\left\langle\eta, \operatorname{Adg} \widehat{\beta}\left(\mathrm{g}^{-1} \cdot \xi\right)\right\rangle
\end{aligned}
$$

so

$$
\widehat{g \cdot \beta}=\operatorname{Adg} \circ \widehat{\beta} \circ \mathrm{g}^{-1} .
$$

Say $\beta$ is invariant if $g \cdot \beta=\beta, \forall g \in G$, then $\beta$ is invariant if and only if $\widehat{\beta}$ intertwines the adjoint and coadjoint representations. Since $G$ is connected, $\beta$ is invariant if and only if the differentiated condition

$$
\beta(X \cdot \xi, \eta)+\beta(\xi, X \cdot \eta)=0, \quad \forall X \in \mathfrak{g}, \xi, \eta \in \mathfrak{g}^{*}
$$

holds. This is equivalent to

$$
\beta^{a b} C_{b d}^{c}+\beta^{c b} C_{b d}^{a}=0
$$

If we define

$$
C_{i}{ }^{j k}=\beta^{j b} C_{i b}{ }^{k}, \quad C^{i}{ }^{k}{ }^{k}=\beta^{i a} C_{a j}{ }^{k}, \quad C^{i j k}=\beta^{i a} \beta^{j b} C_{a b}{ }^{k},
$$

then invariance of $\beta$ implies that $C$ is odd when permuting any upper indices.

If $\beta$ is invariant then the equivariance condition differentiates to give

$$
[X, \widehat{\beta}(\xi)]=\operatorname{adX} \widehat{\beta}(\xi)=\widehat{\beta}(\mathrm{X} \cdot \xi)
$$

so the image $\widehat{\beta}\left(\mathfrak{g}^{*}\right)$ of $\widehat{\beta}$ is an ideal in $\mathfrak{g}$.

We shall consider the Hochschild coboundary operator $\delta$ for the algebra $N$ of smooth functions on a manifold $M$. A $p$-cochain is a $p$-linear map from $N \times \ldots \times N$ ( $p$ copies) to $N$. On $1-$ and 2-cochains $\delta$ is given by

$$
\begin{aligned}
(\delta F)(u, v) & =u F(v)-F(u v)+F(u) v \\
(\delta C)(u, v, w) & =u C(v, w)-C(u v, w)+C(u, v w)-C(u, v) w .
\end{aligned}
$$

We consider only cochains which vanish on the constants. A cochain is called differential if it is given by differential operators on each argument.

It has been shown by Vey [13], that any differential 2-cocycle $C$ can be written as

$$
C=\delta F+B,
$$

where $F$ vanishes on the constants if $C$ does and $B$ is an antisymmetric differential 2-cocycle which is of order 1 in each argument. 


\section{Tangential Star Products}

Let $(M, P)$ be a Poisson manifold with Poisson bracket

$$
\{u, v\}=\langle d u \wedge d v, P\rangle
$$

and set $N=C^{\infty}(M) .(M, P)$ is called regular if $P$ has constant rank. Recall [1] that a star-product on $(M, P)$ is a multiplication map $*: N \times N \rightarrow N[[h]]$

$$
u * v=u v+\sum_{r \geq 1} h^{r} C_{r}(u, v)
$$

such that the binary operation it induces on $N[[h]]$ is associative, has the constant function 1 for identity and the commutator at first order is given by the Poisson bracket

$$
C_{1}(u, v)-C_{1}(v, u)=2\{u, v\} .
$$

We say the star-product is differential if all its cochains are.

We are interested in differential 2-cochains $C(u, v)$ which are tangential to the symplectic leaves of the Poisson manifold and there are a number of ways to define this depending on how close to regular the Poisson structure is. However we shall not need the full strength of tangential, but only require that the 2-cochain does not differentiate any function that is constant on the symplectic leaves. If $\Delta$ is such a function then we say the 2-cochain $C(u, v)$ is $\Delta$-tangential if it vanishes on constants and satisfies

$$
C(\Delta u, v)=\Delta C(u, v)=C(u, \Delta v), \quad \forall u, v \in N .
$$

Lemma 1. A 2-cocycle $C$ is $\Delta$-tangential if and only if

$$
C(1, u)=C(u, 1)=C(\Delta, u)=C(u, \Delta)=0, \quad \forall u \in N .
$$

Proof. Vanishing on constants is common to both conditions. If $C$ is $\Delta$-tangential then $C(\Delta, u)=C(\Delta 1, u)=\Delta C(1, u)=0$ and similarly for the second argument. Conversely, $\delta C=0$ implies that $C(\Delta u, v)=\Delta C(u, v)+C(\Delta, u v)-C(\Delta, u) v=$ $\Delta C(u, v)$ if $C(\Delta, u)=0$ and similarly for the second argument.

Note that this lemma is not true for general 2-cochains. Since coboundaries are cocycles we obtain immediately

Corollary 1. A 2-coboundary $\delta F$ of a 1-cochain $F$ vanishing on constants is $\Delta$ tangential if and only if

$$
F(\Delta u)=\Delta F(u)+F(\Delta) u, \quad \forall u \in N .
$$

Remark 1. If $\delta F$ is $\Delta$-tangential then $F(\Delta)=0$ if and only if $F(\Delta u)=\Delta F(u)$, for all $u \in N$.

Remark 2. If $F(\Delta u)=\Delta F(u)$, for all $u \in N$ and $*$ is a $\Delta$-tangential star-product then the equivalent star-product $*^{\prime}$ defined by

$$
u *^{\prime} v=(1-h F)^{-1}(((1-h F) u) *((1-h F) v))
$$

is also $\Delta$-tangential and the first order term is given by $C_{1}^{\prime}=C_{1}-\delta F$. 
Remark 3. Given any star-product $*$ which is $\Delta$-tangential then $C_{1}$, which is a cocycle from associativity at order 1 , is given by the Poisson bracket plus a coboundary $\delta F$ where $\delta F$ is $\Delta$-tangential. If we show (cf. Lemma 2 below) that $\delta F=\delta F^{\prime}$, where $F^{\prime}(\Delta)=0$ then the star-product $*^{\prime}$ given in Remark 2 is $\Delta$-tangential and its first order term is the Poisson bracket.

In the case where $M=\mathfrak{g}^{*}$ is the dual of a Lie algebra with its coadjoint Poisson structure, then $G$-invariant functions $\Delta$ will be constant on leaves and so satisfy (3).

Lemma 2. Let $F$ be a 1-cochain vanishing on constants whose 2-coboundary $\delta F$ is $\Delta$-tangential where $\Delta$ is an invariant quadratic polynomial on $\mathfrak{g}^{*}$. Then $\delta F=\delta F^{\prime}$ where $F^{\prime}(\Delta)=0$.

Proof. Take linear coordinates $x^{i}$ on $\mathfrak{g}^{*}$ and suppose $F$ is a differential operator of order $k$

$$
F(v)=\sum_{r=1}^{k} A_{i_{1}}^{(r)} i_{r} \partial^{i_{1}}{ }^{i_{r}} v
$$

where the coefficients are smooth and symmetric with respect to permutations of the indices. We have $\delta F(\Delta, v)=0$ and hence,

$$
\sum_{r=2}^{k} r A_{i_{1}}^{(r)} i_{r} \partial^{i_{1}} \Delta \partial^{i_{2}} i^{i_{r}} v+\sum_{r=3}^{k} \frac{r(r-1)}{2} A_{i_{1}}^{(r)} i_{r} \partial^{i_{1} i_{2}} \Delta \partial^{i_{3}} \quad i_{r} v=0
$$

or, relabelling,

$$
\sum_{s=1}^{k-1}(s+1) A_{i_{1} j_{1}}^{(s+1)} j_{s} \partial^{i_{1}} \Delta \partial^{j_{1}} j_{s} v+\sum_{s=1}^{k-2} \frac{(s+1)(s+2)}{2} A_{i_{1} i_{2} j_{1}}^{(s+2)} j_{s} \partial^{i_{1} i_{2}} \Delta \partial^{j_{1}} \quad j_{s} v=0 .
$$

Since $v$ is arbitrary, we must have

$$
\begin{aligned}
A_{i_{1} j_{1}}^{(k)} j_{k-1} & \partial^{i_{1}} \Delta=0 ; \\
A_{i_{1} j_{1} \quad j_{s}}^{(s+1)} \partial^{i_{1}} \Delta+\frac{s+2}{2} A_{i_{1} i_{2} j_{1}}^{(s+2)} j_{s} \partial^{i_{1} i_{2}} \Delta & =0, \quad s=1, \ldots, k-2 ;
\end{aligned}
$$

Now

$$
F(\Delta)=A_{i}^{(1)} \partial^{i} \Delta+A_{i_{1} i_{2}}^{(2)} \partial^{i_{1} i_{2}} \Delta .
$$

We try to replace the second derivatives in (6) by first order terms which we can do recursively using (5). However it is the first of the two terms in (5) which has to be used, and that has the wrong number of derivatives on $\Delta$. When we differentiate this also adds a derivative to the other terms. This means that when we come to the next step we need two derivatives and so on, up to the top term which will need $k-2$ derivatives. The process terminates here since there is no $\partial^{i_{1} i_{2}} \Delta$ term in (4) before differentiating. Proceeding in this way, we reduce all the derivatives in $F(\Delta)$ to first order, say $F(\Delta)=X(\Delta)$. Since this process does not introduce any 0th order terms, it follows $X$ is a derivation. If we now set $F^{\prime}=F-X$ we have the result.

Remark 4. It is worth observing that the process for constructing the derivation $X$ in the above proof is the same for all quadratic functions $\Delta$ so that $X$ depends only on $F$ and not on $\Delta$. 
As a consequence of this lemma and Remark 3, in studying $\Delta$-tangential starproducts on $\mathfrak{g}^{*}$ for invariant quadratic forms $\Delta$ we are free to assume that the first order term is the Poisson bracket.

Lemma 3. Let $A_{i j k} \in C^{\infty}(U)$, where $U$ is a star-shaped neighbourhood of 0 in $\mathfrak{g}^{*}$, symmetric with respect to permutations of all its indices. If $\beta$ is an invariant symmetric bilinear form on $\mathfrak{g}^{*}$ and

$$
x_{r} \beta^{r k} A_{i j k}=x_{r} x_{s} \beta^{a b} C_{a i}{ }^{r} C_{b j}{ }^{s}
$$

on $U$ then $\widehat{\beta}\left(\mathfrak{g}^{*}\right)$ is two-step nilpotent: $\left[\widehat{\beta}\left(\mathfrak{g}^{*}\right),\left[\widehat{\beta}\left(\mathfrak{g}^{*}\right), \widehat{\beta}\left(\mathfrak{g}^{*}\right)\right]\right]=0$.

Proof. Since $U$ is star-shaped with respect to $0, A_{i j k}$ has a Taylor expansion at 0 to any order. Let $a_{i j k}{ }^{l} x_{l}$ be the linear term, then comparing quadratic terms in (7) we have

$$
x_{r} \beta^{r k} a_{i j k}{ }^{s} x_{s}=x_{r} x_{s} \beta^{a b} C_{a i}{ }^{r} C_{b j}{ }^{s},
$$

hence

$$
\beta^{r k} a_{i j k}{ }^{s}+\beta^{s k} a_{i j k}{ }^{r}=\beta^{a b} C_{a i}{ }^{r} C_{b j}{ }^{s}+\beta^{a b} C_{a i}{ }^{s} C_{b j}{ }^{r} .
$$

If we define

$$
\widehat{a}^{a b c d}=\beta^{a i} \beta^{b j} \beta^{c k} a_{i j k}{ }^{d},
$$

then we have, using the symmetry of $a$ in its first three indices,

$$
\widehat{a}^{r i j s}+\widehat{a}^{s i j r}=\beta^{a b} C_{a}{ }^{i r} C_{b}{ }^{j s}+\beta^{a b} C_{a}{ }^{i s} C_{b}{ }^{j r}
$$

using (2).

Interchanging $r$ and $i$ in (8) we have

$$
\widehat{a}^{r i j s}+\widehat{a}^{s r j i}=-\beta^{a b} C_{a}{ }^{i r} C_{b}{ }^{j s}+\beta^{a b} C_{a}{ }^{r s} C_{b}{ }^{j i} .
$$

Now

$$
\begin{aligned}
\beta^{a b} C_{a}{ }^{r s} C_{b}{ }^{j i} & =-\beta^{a s} C_{a}{ }^{r b} C_{b}{ }^{j i} \\
& =-\beta^{a s} \beta^{c r} \beta^{d j} C_{a c}{ }^{b} C_{b d}{ }^{i} \\
& =\beta^{a s} \beta^{c r} \beta^{d j}\left(C_{c d}{ }^{b} C_{b a}{ }^{i}+C_{d a}{ }^{b} C_{b c}{ }^{i}\right) \\
& =\beta^{a b} C_{b}{ }^{j r} C_{a}{ }^{i s}-\beta^{a b} C_{b}{ }^{j s} C_{a}{ }^{i r},
\end{aligned}
$$

hence (9) becomes

$$
\widehat{a}^{r i j s}+\widehat{a}^{s r j i}=-2 \beta^{a b} C_{a}{ }^{i r} C_{b}{ }^{j s}+\beta^{a b} C_{a}{ }^{i s} C_{b}{ }^{j r} .
$$

Adding twice Eq. (8) to Eq. (10) gives

$$
3 \widehat{a}^{r i j s}+2 \widehat{a}^{s i j r}+\widehat{a}^{s r j i}=3 \beta^{a b} C_{a}^{i s} C_{b}{ }^{j r} .
$$

The right-hand side of this equation is skew symmetric in $j$ and $r$, so taking the skew part of the left-hand side gives

$$
\widehat{a}^{s i j r}-\widehat{a}^{s i r j}=3 \beta^{a b} C_{a}^{i s} C_{b}^{j r} .
$$

But the right-hand side is also skew in $i$ and $s$ and the left-hand side is now symmetric in $i$ and $s$, so both must vanish. Thus 


$$
0=\beta^{a b} C_{a}{ }^{i s} C_{b}{ }^{j r}=-\beta^{a s} C_{a}{ }^{i b} C_{b}{ }^{j r} .
$$

Contracting with $X_{r}$ gives

$$
\beta^{a s} \beta^{i c} \beta^{j d}\left[\left[X_{a}, X_{c}\right], X_{d}\right]=0
$$

which is the required condition.

In the coordinates $x_{1}, \ldots, x_{n}$ the Poisson structure on $C^{\infty}\left(\mathfrak{g}^{*}\right)$ is given by

$$
\{u, v\}=x_{r} C_{i_{1} j_{1}}^{r} \partial^{i_{1}} u \partial^{j_{1}} v .
$$

Define a 2-cochain on $C^{\infty}\left(\mathfrak{g}^{*}\right)$ by

$$
\begin{aligned}
C(u, v)= & \frac{1}{2} x_{r} x_{s} C_{i_{1} j_{1}}{ }^{r} C_{i_{2} j_{2}}{ }^{s} \partial^{i_{1} i_{2}} u \partial^{j_{1} j_{2}} v \\
& \quad-\frac{1}{3} x_{r} C_{i_{1} j_{1}}{ }^{s} C_{s i_{2}}{ }^{r}\left(\partial^{i_{1} i_{2}} u \partial^{j_{1}} v+\partial^{j_{1}} u \partial^{i_{1} i_{2}} v\right) .
\end{aligned}
$$

Up to a normalization this is the order 2 term in the star product coming from the enveloping algebra via Poincaré-Birkhoff-Witt. We leave the routine verification of the following lemma to the reader.

\section{Lemma 4.}

$$
\delta C(u, v, w)=\{\{u, w\}, v\}
$$

Proposition 1. Let $\mathfrak{g}$ be a Lie algebra, $\beta$ an invariant bilinear form on $\mathfrak{g}^{*}$, and $\Delta \in$ $C^{\infty}\left(\mathfrak{g}^{*}\right)$ be defined as above. If there is a differential 2-cochain $D(u, v)$ on $C^{\infty}(U)$, where $U$ is a neighbourhood of 0 in $\mathfrak{g}^{*}$, and satisfying

(i) $\quad D(\Delta, v)=0, \quad \forall v \in C^{\infty}(U)$;

(ii) $\delta D(u, v, w)=\{\{u, w\}, v\}$,

then $\widehat{\beta}\left(\mathfrak{g}^{*}\right)$ is two-step nilpotent.

Proof. Shrinking $U$ if necessary we may assume it is star-shaped with respect to 0 . We show that the existence of such a cochain $D$ implies the existence of functions $A_{i j k}$ satisfying the conditions of Lemma 3.

By condition (ii) and Lemma 4 it follows that $\delta(D-C)=0$ hence $D-C$ has the form

$$
D-C=\delta T+B
$$

where $B$ is a 2-cocycle involving differential operators of order $(1,1)$ and $T$ is an arbitrary 1-cochain. From condition (i) it follows that

$$
C(\Delta, v)+\delta T(\Delta, v)+B(\Delta, v)=0, \quad \forall v \in C^{\infty}(U)
$$

and hence that $C(\Delta, v)+\delta T(\Delta, v)$ is a differential operator on $v$ of order 1 . Let $k$ be the order of $T$, then $\delta T(\Delta, v)$ has order $k-1$ whilst 


$$
\begin{aligned}
C(\Delta, v)= & \frac{1}{2} x_{r} x_{s} C_{i_{1} j_{1}}{ }^{r} C_{i_{2} j_{2}}{ }^{s} 2 \delta_{a}^{i_{1}} \delta_{b}^{i_{2}} \beta^{a b} \partial^{j_{1} j_{2}} v \\
& \quad-\frac{1}{3} x_{r} C_{i_{1} j_{1}}{ }^{s} C_{s i_{2}}{ }^{r} 2 \delta_{a}^{i_{1}} \delta_{b}^{i_{2}} \beta^{a b} \partial^{j_{1}} v-\frac{1}{3} x_{r} C_{i_{1} j_{1}}{ }^{s} C_{s i_{2}}{ }^{r} 2 \beta^{j_{1} b} x_{b} \partial^{i_{1} i_{2}} v \\
= & x_{r} x_{s} C_{a j_{1}}{ }^{r} C_{b j_{2}}{ }^{s} \beta^{a b} \partial^{j_{1} j_{2}} v \\
& \quad-\frac{2}{3} x_{r} x_{s} C_{j_{2} b}{ }^{a} C_{a j_{1}}{ }^{r} \beta^{s b} \partial^{j_{1} j_{2}} v-\frac{2}{3} x_{r} C_{a j_{1}}{ }^{s} C_{s b}{ }^{r} \beta^{a b} \partial^{j_{1}} v \\
= & x_{r} x_{s} C_{a j_{1}}{ }^{r} C_{b j_{2}}{ }^{s} \beta^{a b} \partial^{j_{1} j_{2}} v \\
& \quad+\frac{2}{3} x_{r} x_{s} C_{j_{2} b}{ }^{s} C_{a j_{1}}{ }^{r} \beta^{a b} \partial^{j_{1} j_{2}} v-\frac{2}{3} x_{r} C_{a j_{1}}{ }^{s} C_{s b}{ }^{r} \beta^{a b} \partial^{j_{1}} v \\
= & \frac{1}{3} x_{r} x_{s} C_{a j_{1}}{ }^{r} C_{b j_{2}}{ }^{s} \beta^{a b} \partial^{j_{1} j_{2}} v-\frac{2}{3} x_{r} C_{a j_{1}}{ }^{s} C_{s b}{ }^{r} \beta^{a b} \partial^{j_{1}} v .
\end{aligned}
$$

Hence

$$
\delta T(\Delta, v)+\frac{1}{3} x_{r} x_{s} C_{a j_{1}}{ }^{r} C_{b j_{2}}{ }^{s} \beta^{a b} \partial^{j_{1} j_{2}} v
$$

is a differential operator in $v$ of order 1 .

We write $T$ as

$$
T(v)=\sum_{r=0}^{k} A_{i_{1}}^{(r)} i_{r} \partial^{i_{1}} \quad i_{r} v,
$$

where the coefficients are smooth functions on $U$, symmetric with respect to permutations of the indices. Considering terms of order 2 or greater in (11) we have

$$
\begin{aligned}
& \sum_{r=3}^{k} r A_{i_{1}}^{(r)}{ }_{i_{r}} \partial^{i_{1}} \Delta \partial^{i_{2}} \quad{ }^{i_{r}} v+ \\
& \quad \sum_{r=4}^{k} \frac{r(r-1)}{2} A_{i_{1}}^{(r)} i_{r} \partial^{i_{1} i_{2}} \Delta \partial^{i_{3} \quad i_{r}} v=\frac{1}{3} x_{p} x_{q} C_{a j_{1}}{ }^{p} C_{b j_{2}}{ }^{q} \beta^{a b} \partial^{j_{1} j_{2}} v
\end{aligned}
$$

so that

$$
\begin{gathered}
A_{i_{1} \quad i_{r}}^{(r)} \partial^{i_{1}} \Delta+\frac{(r-1)}{2} A_{i_{1}}^{(r)} i_{r} \partial^{i_{1} i_{2}} \Delta=0, \quad r \geq 4, \\
A_{i_{1} j_{1} j_{2}}^{(3)} \partial^{i_{1}} \Delta+A_{i_{1} i_{2} j_{1} j_{2}}^{(4)} \partial^{i_{1} i_{2}} \Delta=\frac{1}{3} x_{p} x_{q} C_{a j_{1}}{ }^{p} C_{b j_{2}}{ }^{q} \beta^{a b} .
\end{gathered}
$$

The argument now follows the same lines as in Lemma 2: we express the terms $A_{i_{1} i_{2} j_{1} j_{2}}^{(4)} \partial^{i_{1} i_{2}} \Delta$ in (13) as first order operators acting on $\Delta$ using Eq. (12) recursively. Renaming the left-hand side as $\frac{3}{2} A_{i_{1} j_{1} j_{2}} \partial^{i_{1}} \Delta$ this will satisfy

$$
\frac{1}{2} A_{i_{1} j_{1} j_{2}} \partial^{i_{1}} \Delta=x_{r} x_{s} C_{a j_{1}}{ }^{r} C_{b j_{2}}{ }^{s} \beta^{a b}
$$

where $A_{i j k}$ is completely symmetric. Since $\partial^{i_{1}} \Delta=2 x_{r} \beta^{r i_{1}}$ we have a smooth solution of (7) on a neighbourhood of the origin and so can apply Lemma 3 to give the result. 
This proposition yields immediately the following theorem:

Theorem 2. If $\mathfrak{g}$ is a Lie algebra with a star-product on a neighbourhood of 0 in $\mathfrak{g}^{*}$ which is both differential and tangential to the coadjoint orbits then $\widehat{\beta}\left(\mathfrak{g}^{*}\right)$ is two-step nilpotent for every invariant bilinear form $\beta$ on $\mathfrak{g}^{*}$.

Proof. An invariant bilinear form will yield a quadratic form $\Delta$ which is constant on each coadjoint orbit so the cochains of the tangential star-product are $\Delta$-tangential. If necessary we replace the given star-product by one whose first order term is the Poisson bracket and which is also $\Delta$-tangential. The second order term in the starproduct will now satisfy the conditions of the proposition.

Corollary 2. If $\mathfrak{g}$ is a semisimple Lie algebra there is no differential star-product on any neighbourhood of the origin in $\mathfrak{g}^{*}$ which is tangential to the coadjoint orbits.

Proof. If such a star-product existed it would be $\Delta$-tangential where $\beta$ is the Killing form and $\Delta$ the associated quadratic form on $\mathfrak{g}^{*}$. Since the Killing form of a semisimple Lie algebra is non-degenerate the image of $\widehat{\beta}$ is the whole of $\mathfrak{g}$. Since $\mathfrak{g}$ cannot be both semisimple and two-step nilpotent, no tangential star-product can exist.

Remark 5. If $\mathfrak{g}$ is two-step nilpotent, the star-product on $\mathfrak{g}^{*}$ induced by the linear isomorphism between the symmetric algebra $S(\mathfrak{g}) \subset C^{\infty}\left(\mathfrak{g}^{*}\right)$ and the universal enveloping algebra $U(\mathfrak{g})$ given by total symmetrization is tangential and differential.

Let $\beta$ be an invariant bilinear form on $\mathfrak{g}^{*}$ and $\widehat{\beta}: \mathfrak{g}^{*} \rightarrow \mathfrak{g}$ the corresponding equivariant map. We have observed that the image $\mathfrak{h}=\widehat{\beta}\left(\mathfrak{g}^{*}\right)$ is an ideal in $\mathfrak{g}$. It is also clear that there is a well-defined bilinear form $\bar{\beta}$ on $\mathfrak{h}$ such that

$$
\bar{\beta}(\widehat{\beta}(\xi), \widehat{\beta}(\eta))=\beta(\xi, \eta)
$$

since $\beta$ and $\widehat{\beta}$ have the same kernel $K \subset \mathfrak{g}^{*}$. It is equally clear that $\bar{\beta}$ is invariant under all the automorphisms of $\mathfrak{h}$ induced by the adjoint action of $G$. Moreover, $K$ is the annihilator of $\mathfrak{h}$ in $\mathfrak{g}^{*}$.

Conversely, let $\mathfrak{g}$ be a Lie algebra with $\mathfrak{h}$ an ideal and $\bar{\beta}$ a $G$-invariant nondegenerate bilinear form on $\mathfrak{h}$. Then $\bar{\beta}$ induces a bilinear form $\bar{\beta}^{*}$ on $\mathfrak{h}$ * which can be extended to $\mathfrak{g}^{*}$ by zero on the annihilator of $\mathfrak{h}$ :

$$
\beta(\xi, \eta)=\bar{\beta}^{*}\left(\left.\xi\right|_{\mathfrak{h}},\left.\eta\right|_{\mathfrak{h}}\right)
$$

It is easy to check that $\beta$ is $G$-invariant.

So we get a 1-1 correspondence between $G$-invariant bilinear forms on $\mathfrak{g}^{*}$ and $G$-invariant non-degenerate bilinear forms on ideals in $\mathfrak{g}$.

Thus the algebras which are of interest are two-step nilpotent and have a nondegenerate invariant bilinear form. We do not know of a classification of these Lie algebras. To show that we can not in general reduce the problem any further we observe that we can obtain examples of such algebras by taking a Lie algebra $k$ without centre and having a nondegenerate invariant bilinear form $\beta_{0}$. We set $\mathfrak{g}=\mathfrak{k} \oplus \mathfrak{k}$ as vector spaces with bracket

$$
\left[\left(X, X^{\prime}\right),\left(Y, Y^{\prime}\right)\right]=(0,[X, Y])
$$

and bilinear form

$$
\beta\left(\left(X, X^{\prime}\right),\left(Y, Y^{\prime}\right)\right)=\beta_{0}\left(X, Y^{\prime}\right)+\beta_{0}\left(X^{\prime}, Y\right) .
$$

The invariance of $\beta$ is equivalent to the invariance of $\beta_{0}$. 
Remark 6. Let $\mathfrak{k}$ be a compact semisimple Lie algebra, $\mathfrak{g}$ its complexification and $\mathfrak{g}=\mathfrak{k}+\mathfrak{a}+\mathfrak{n}$ a Iwasawa decomposition when viewed as a real Lie algebra, where $i \mathfrak{a}$ is a maximal toral subalgebra of $\mathfrak{k}$. In [8] Lu and Weinstein showed that $(\mathfrak{g}, \mathfrak{k}, \mathfrak{a}+\mathfrak{n})$ has the structure of a Manin triple. This gives the simply connected Lie group $A N$ a Poisson Lie group structure whose Poisson tensor has linear part at the identity element which is isomorpphic to the coadjoint Poisson structure on $\mathfrak{k}^{*}$. Since $\mathfrak{k}$ is compact semisimple, it follows from a result of Conn [4] that the Poisson structure on $A N$ is linearizable in a neighbourhood of the identity element. In fact, in [9] Ginzburg and Weinstein have shown that the Poisson structures on $\mathfrak{k}^{*}$ and $A N$ are globally diffeomorphic, a result which was conjectured by Lu and Ratiu in [7]. Thus any tangential differential star product on $A N$ which is tangential to the symplectic leaves would induce a tangential differential star product on $\mathfrak{k}^{*}$ and as we have seen that is not possible for semisimple $\mathfrak{k}$. Hence the groups $A N$ with their Poisson structure coming from the Iwasawa decomposition give examples of Poisson Lie groups which have no differential star product tangential to the symplectic leaves.

A similar argument does not apply to the Poisson Lie structure on $K$ since we know from [3] that if $K$ is not a product of copies of $S U(2)$ then its Poisson Lie structure is not isomorphic near the identity element to a neighbourhood of the origin in $(\mathfrak{a}+\mathfrak{n})^{*}$. Not only that, but it is not difficult to show (using Remark 5) that $(\mathfrak{a}+\mathfrak{n})^{*}$ has no invariant bilinear forms, so our approach imposes no conditions on tangential deformations. Thus it is an open question whether or not there is a differential star product on $K$ or on $(\mathfrak{a}+\mathfrak{n})^{*}$ which is tangential to the symplectic leaves.

\section{References}

1 Bayen, F, Flato, M, Fronsdal, C, Lichnerowicz, A, Sternheimer, D : Deformation theory and quantization Lett Math Phys 1, 521-530 (1977)

2 Bayen, F, Flato, M, Fronsdal, C, Lichnerowicz, A, Sternheimer, D : Deformation theory and quantization Ann Phys. 111, 61-110 (1978)

3 Cahen, M, Gutt, S, Rawnsley, J : Non-linearisability of the Iwasawa Poisson Lie structure Lett Math Phys 24, 79-83 (1992)

4 Conn, J F : Normal forms for smooth Poisson structures Ann Math 121, 565-593 (1985)

5 De Wilde, M., Lecomte, P B : Existence of star-products and of formal deformations of the Poisson Lie algebra of arbitrary symplectic manifolds Lett Math Phys 7, 487-496 (1983)

6 Fedosov, B V : A simple geometrical construction of deformation quantization $\mathrm{J}$ Diff Geom 40, 213-238 (1994)

7 Lu, J H , Ratiu, T : On the nonlinear convexity theorem of Kostant J Am Math. Soc 4, 349-363 (1991)

$8 \mathrm{Lu}, \mathrm{J} \mathrm{H}$, Weinstein, A : Poisson-Lie groups, dressing transformations and Bruhat decompositions $\mathrm{J}$ Diff Geom. 31, 501-526 (1990)

9 Ginzburg, V.L, Weinstein, A.: Lie--Poisson structure on some Poisson Lie groups J Am Math Soc 5, 445-453 (1992)

10 Masmoudi, M : Tangential formal deformations of the Poisson bracket and tangential star products on a regular Poisson manifold. J Geom Phys 9, 155-171 (1992)

11 Omori, H, Maeda, Y, Yoshioka, A : Weyl manifolds and deformation quantization Adv Math 85, 224-255 (1991)

12 Omori, H, Maeda, Y, Yoshioka, A : Deformation quantization of Poisson algebras Contemp Math 179, 213-240 (1994)

13 Vey, J : Déformation du crochet de Poisson sur une variété symplectique. Comment Math Helvet 50, 421-454 (1975)

14 Weinstein, A : The local structure of Poisson manifolds J Diff Geom 18, 523-557 (1983)

Communicated by H. Araki 